\title{
Advanced Analysis Method for Human Relationship Based on Fuzzy Theory
}

\author{
Toshihiro YOSHIZUMI*1 • Tomoo SUMIDA*2 • Yasunori SHIONO*3 • \\ Mitsuhiro NAMEKAWA*4 $\cdot$ Kensei TSUCHIDA*2
}

\begin{abstract}
In this paper, we proposed advanced analytical method so that various analysis is possible. In the past, various analysis methods for human relationships have been proposed. Moreno proposed sociometry analysis and Yamashita et al. proposed an analysis method applying fuzzy graphs to it. Yamashita's method is to analyze human relationships based on the partition tree. As preconditions for analysis, the nodes belonging to the same similarity in the partition tree are analyzed. In this paper, we provide a method that can analyze nodes belonging to any degree of similarity. Also, depending on the purpose of the analyst, a directed graph, such as a sociogram, may be more effective, or an undirected graph may be more effective. Therefore, there are both merits and demerits to using either method. We also aim to provide a new foundation that can analyze both of the two analysis methods.
\end{abstract}

Keywords: analysis for human relationship, sociometry, sociogram, fuzzy theory, partition tree

\section{Introduction}

In the past, various analysis methods for human relationships have been proposed. Sociometry analysis was proposed by Moreno [1]. In the sociometry analysis, the analyst conducts a questionnaire survey and analyzes based on the survey results from the subjects selected. The results are represented by a diagram called a sociogram. A sociogram is a graph representation of the obtained data. An analyst analyzes sociograms. The method used for analysis differs depending on the purpose of how the analyst wants to grow the group. In the sociometry analysis, one result (sociogram) is obtained for one question. Therefore, the sociogram reflects the superficial consciousness of the subject. As a result, the sociometry analysis revealed how the subjects think about their partner. Yamashita et al. developed analysis methods applying fuzzy theory to Moreno's sociometry analysis [2-7]. There are differences between the two methods. Sociometry analysis analyzed the one-way relationship between subjects. On the other hand, Yamashita's analysis method analyzed the mutual relationship between subjects. Also, in sociometry analysis, one sociogram is obtained from one question. On the other hand, Yamashita's method consolidated the results obtained from multiple questions into one fuzzy graph. As a result, sociometry analysis revealed superficial consciousness, whereas Yamashita's method revealed the subconscious mind of the subject indirectly by consolidating multiple question results. The edge value is 0 (non-connection) or 1 (connection) in the sociogram, whereas it

* 1 Graduate School of Engineering, Toyo University

*2 Faculty of Information Sciences and Arts, Toyo University

*3 Organization for Information Strategy and Promotion, Yokohama National University

*4 Graduate School of Business Innovation, Kaetsu University is between 0.0 and 1.0 in the fuzzy graph.

Yamashita's method provided basic methods for automatically analyzing subgroups, opinion leaders, and isolated members. However, human relationships are complex, and analysts are not satisfied only with the results obtained from a quantitative analysis method. For this reason, the method used for analysis differs depending on the purpose of an analyst. Depending on the purpose of the analyst, a directed graph like the sociogram may be more effective or the undirected graph may be more effective. Therefore, there are both merits and demerits to using either method. Therefore, we aim to provide a new foundation that can analyze both of the two analysis methods.

Also, Yamashita's method analyzes the human relations based on the partition tree. As a precondition for the analysis, only the nodes belonging to the same similarity level in the partition tree are targets for analysis. Also, the optimal degree of similarity is calculated for which similarity is to be analyzed, and only the optimal similarity is the target of analysis [6]. We also aim to eliminate preconditions and provide a method that can analyze nodes belonging to any degree of similarity.

This paper is organized as follows: in Section 2 we introduce the analysis method for the human relationships, while Section 3 explain the fuzzy model for human relationships. In Section 4, we propose the advanced analysis method for group relationships in human relationships and describe various analysis indices for fuzzy graph. Section 5 presents a case study and the description of some results obtained through the proposed method. Finally, conclusions and plans for future work are presented in Section 6.

\section{Analysis Method for Human Relationships}

Various analysis methods for human relationship have been proposed. In this section, we introduce existing analysis methods 


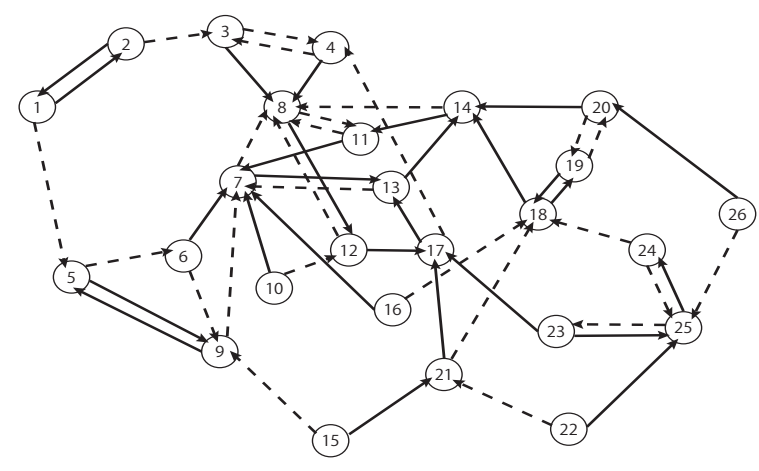

Fig. 1 An example of sociogram.

and their problems.

\subsection{Sociometry Analysis}

Sociometry analysis is a measurement method proposed by Moreno as a method of measuring and evaluating the group structure of human relations using tables and figures [1]. Human society consists of mutually related groups and the group consists of social, economic and cultural ties between individuals. Sociometry analysis focuses on structural analysis in smaller groups, for example, classrooms and workplaces, rather than society as a whole. For analysis, analyst use data obtained by asking people to be surveyed, "Who will you choose as a friend (or colleague, advisor, etc.)?" A graph representation of the obtained data is called a sociogram. In the sociogram, individuals are represented as nodes and selective relationships are represented by an arc.

An example of sociogram is shown Fig. 1 [8]. Fig. 1 shows a relationship between the first and second selected persons, who selected their preferred partners for dinner. The solid line represents the first and the dotted line represents the second selected persons. From the sociogram shown in Figure1, we can see the state of the groups, such as those who concentrate on selections from other people, or those who make mutual choices.

\subsection{Observation Method and Interview Method for Human Relationships}

In analyzing human relationships, the most appropriate method for grasping the boundaries of peer group membership and analyzing internal structure is the observation and interview method [9]. In the observation method, the analyst observes the activity of the group for a certain period and confirms the existence of the group by direct external observation. Then, analysts interview each member and check the group boundaries.

From the results of the survey using the observation and interview method, it is possible to analyze the internal structure of the peer group as shown below.

- The moment and timing of group formation

- The purpose of the group

- The moment of joining the group

- Emotional relationship to other members

\subsection{Problems of Existing Analysis Methods}

In the sociometry analysis, it is possible to analyze and eval- uate the group structure with only data obtained from simple questions. Sociometric analysis is a method of measuring and analyzing human relations and group structure. Therefore, it is not desirable to grasp the group itself or to clarify the boundaries of the group membership.

Analysis by the observation and interview method allows more detailed analysis than sociometry analysis such as analysis of inner group structure. On the other hand, the fact that it takes a long time to investigate is a demerit. In a research study on the structure of a peer group of children by Sumida [9], it required approximately 2 weeks to obtain the results of the observation method investigation, and about 5.5 months to obtain results from the survey using the interview method. It takes a long time just for the survey, and presumably, even more time to include analysis. Also, in analysis using the observation and interview method, it is difficult to analyze unless the analyst possesses sophisticated knowledge, such as a specialist in sociology. Other methods include extracting interpersonal relationships using motion picture processing [10]. In this method, it is necessary to prepare places and equipment to be recorded as motion pictures, and it is necessary to consider that the psychological state that results from being recorded affects subject's behavior.

On the other hand, in human relations analysis applying fuzzy graphs, it is possible to analyze human relationships in a short time using a small question survey.

\section{Fuzzy Model for Human Relationships}

In this section, we explain the fuzzy model for human relationship that can be quantitatively analyzed [2-7].

\section{1 Fuzzy Graph Model}

Human relationships within the group can be analyzed using data obtained from a simple questionnaire. It is possible to analyze human relationships rationally and more clearly within the group by constructing fuzzy models from results obtained from a simple questionnaire and applying fuzzy clustering and fuzzy similarity.

\subsection{Response Table and Response Matrix}

If we ask on the M questionnaires to L students: "Write your friendly $\mathrm{N}(1 \leq N \leq L)$ members in the order of those with whom you would like to do activities $\operatorname{Ap}(1 \leq p \leq M)$," then we have a response table. Analyzing this table, we have the response matrix $K^{(p)}=\left(k_{i j}^{(p)}\right) \cdot k_{i j}^{(p)}$ is the ranking value $k(0 \leq k \leq N)$ in which a student $S_{i}$ has selected another student $S_{j}(i, j=1, \ldots, L)$. However, $k=n-m(0 \leq k \leq N), m=$ (number of order) -1 for each activity and $n$ is defined as follows.

$$
n=\left[\frac{\sum n_{i}}{M L}+0.5\right]
$$

Where $n_{i}$ is the sum of numbers by which $S_{i}$ selects his friendly members for $M$ activities and [ ] is a gaussian symbol.

Examples of questions are as follows.

- Please write your $N$ friends in the order of those with whom 
(A1) you would like to study in group

(A2) you would like to take lunch together

(A3) you would like to do club activities

(A4) you would like to do volunteer activities

\subsection{Evaluation Matrix}

Analyzing the matrix $K^{(p)}=\left(k_{i j}^{(p)}\right)$, we could define the evaluation matrix $R=\left(r_{i j}\right)$ as follows.

$$
r_{i j}=\sum_{p}\left(n-k_{i j}^{(p)}+1\right)
$$

\subsection{Fuzzy Matrix and Fuzzy Graph}

Analyzing the matrix $R=\left(r_{i j}\right)$, we could define the fuzzy degree of "friendliness" and that of "preference" among the members within the group. Let $f_{i j}=r_{i j} / n M$, and we consider $f_{i j}$ as the degree of fuzziness to which a member $S_{i}$ prefers the member $S_{j}$. We have a fuzzy cluster matrix $F=\left(f_{i j}\right), 0 \leq f_{i j} \leq 1$, where $f_{i j}=1$ if $i=j$.

On the other hand, a fuzzy graph is defined mathematically as $G=(V, F)$, where $V=\left\{v_{i}\right\}$ is the set of nodes and $F=\left(f_{i j}\right)$ is the relation matrix; where $f_{i j}$ is the value of the arc from $v_{i}$ to $v_{j}$. A graph is called a fuzzy graph if its value is between 0.0 and 1.0. The fuzzy matrix $F$ is equivalent to a fuzzy graph. As observed above, we could build a fuzzy model representing the connective structure among the members in a group.

\subsection{Cluster Analysis for Fuzzy Graph}

Let $2 / s_{i j}=1 / f_{i j}+1 / f_{j i}$, and we consider $s_{i j}$ as the degree of the fuzziness to which two members $S_{i}$ and $S_{j}$ are mutually friendly. Thus, we have the fuzzy matrix $S=\left(s_{i j}\right), 0 \leq s_{i j} \leq 1$, and $s_{i j}=0$ if $f_{i j} f_{j i}=0$. Here, $s_{i j} \sim 1$ means that $S_{i}$ and $S_{j}$ are very friendly, and $s_{i j} \sim 0$ means that they are not very friendly. In order to analyze the similarity structure of nodes for a fuzzy graph, we use the symmetric relational matrix $S=\left(s_{i j}\right)$ and compute it for transitive closure $\widehat{S}=\left(\widehat{s_{i j}}\right)$. After calculating transitive closure of the symmetric relational matrix, we define the c-cut matrix as follows.

$$
S^{c}=\left(s_{i j}^{c}\right), s_{i j}^{c}= \begin{cases}1, & \text { for } \widehat{s_{i j}} \geq \mathrm{c} \\ 0, & \text { for } \widehat{s_{i j}}<\mathrm{c}\end{cases}
$$

The relation $R_{c}$ of the c-cut matrix $S^{c}$ gives a similarity of relations among nodes. Thence, we have the partition tree (dendrogram) by changing level $c$ of the c-cut matrix, which represents the clustering situation of nodes in a fuzzy graph. A value of level $c$ is defined by an element of the transitive closure. Each level $c$ is defined by one of the elements in the transitive closure. We call it a similarity level $R_{c}$ or, hereafter, a level c. The partition tree is produced by analyzing transitive closure as shown in Fig. 2. In this way, partition trees are produced by cluster analysis using $\widehat{S}$ and its c-cut matrices and show the clustering situation and the branching process among members.

\section{Advanced Analysis Method for Group Relationships in Human Relationships}

In this section, we define the relationship between groups, or groups and individuals. In addition, we propose the advanced analysis method for group relations based on fuzzy graph and describe various indices for analyzing fuzzy graph.

\subsection{Analysis Index of Human Relationships Applying the Fuzzy Graph}

There are various indices for analyzing graphs and networks. This refers indicators that are considered to be the minimum necessary for analysis of human relationships [11]. Some analytical indicators are applied to fuzzy graphs and their definitions extended, and we show them as indicators of human relationship analysis. The target fuzzy graph to be analyzed is defined mathematically as $G=(V, S)$, where $V=\left\{v_{i}\right\}$ is a set of nodes and $S=\left(s_{i j}\right)$ is a symmetric relation matrix; where $s_{i j}$ is the value of the edge between $v_{i}$ and $v_{j}$.

\subsubsection{Fuzzy Graph Density}

This is an indicator of closeness and complexity in a relationship. The existing index is the number of links divided by the number of links when all nodes are connected. Generally, edge values of graphs and networks are represented by 0 (no connection) or 1 (connection). However, in fuzzy graphs, edge values range from 0.0 to 1.0 . Therefore, the fuzzy density $(F D)$ considering the edge value is defined as follows.

$$
F D=\frac{\sum_{v_{i}, v_{j} \in V} s_{i j}}{|V \times V|}
$$

\subsubsection{Distance between Nodes}

This is calculated the distance between two specific nodes. There are indices such as shortest, longest, and total route average. There is also a method of using the number of edges as a distance and a method of calculating the value of edges. In case of having a value on an edge like a fuzzy graph, the shortest path can be calculated by Dijkstra's method, Thorup's algorithm, etc.

\subsubsection{Node Reachability}

This identifies that there is a reachable route from one node to another node. It is possible to identify an isolated member (IS) not belonging to a group in a human relationship by the following formula.

$$
I S=\min _{1 \leq i \leq n}\left\{\sum_{j}^{n} s_{i j}\right\}
$$

\subsubsection{Degree Centrality}

Measures centrality from the number of edges connected to the node and identifies the node with the widest relationship to other nodes. Degree Centrality $(D C)$ is defined as follows.

$D C=\max (d(v))$ 


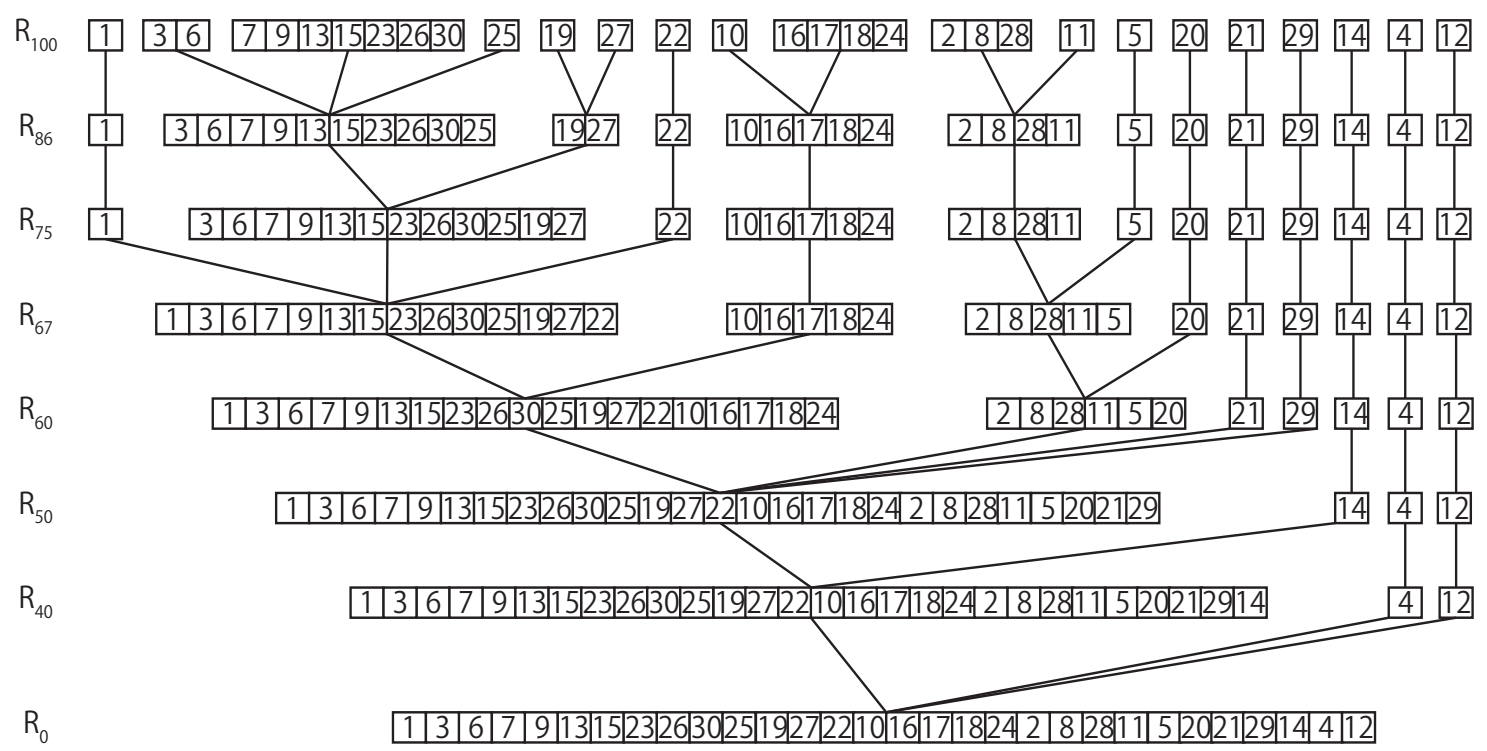

Fig. 2 Partition tree from the question survey results.

\subsubsection{Relationship Centrality}

Measures centrality from the similarity of the edge connected to the node and identifies the node with strong influence. Relationship Centrality $(O P)$ is defined as follows.

$$
O P=\max _{1 \leq i \leq n}\left\{\sum_{j}^{n} s_{i j}\right\}
$$

Furthermore, by analyzing the fuzzy graph of $G=(V, F)$, it is also possible to identify the node that most affects its surroundings as follows.

$$
O P=\max _{1 \leq i \leq n}\left\{\sum_{j}^{n} f_{i j}\right\}
$$

\subsubsection{Relationship Betweenness Central- ity [12]}

In relationship betweeness centrality $(R B C)$, when there is spread of information and influence between two clusters, which node should be mediated is specified. $R B C$ is obtained from the maximum value of the sum of the edge values from the nodes of one cluster to nodes belonging to another cluster between any two clusters.

$$
\begin{aligned}
& v_{i} \in C L_{A},\left|C L_{A}\right|=m \\
& v_{j} \in C L_{B},\left|C L_{B}\right|=n \\
& R B C=\max _{1 \leq i \leq m}\left\{\sum_{j}^{n} s_{i j}\right\}
\end{aligned}
$$

$R B C$ is specified from $C L_{A}$ to $C L_{B}$ in Fig. 3. At this time, $A_{1}=0.2, A_{2}=0.3+0.4=0.7$, and $A_{3}=0.5$. Therefore, it can be seen that it is better to mediate the node $A_{2}$ with the maximum value 0.7. Similary, $R B C$ is specified from $C L_{B}$ to $C L_{A}$. At this time, $B_{1}=0.2+0.3=0.5$ and $B_{2}=0.4+0.5=0.9$. Therefore, it can be seen that it is better to mediate the node $B_{2}$ with the maximum value 0.9 .

\subsection{Analysis Method for Group Relationship}

Previous studies on what kind of group existed have been

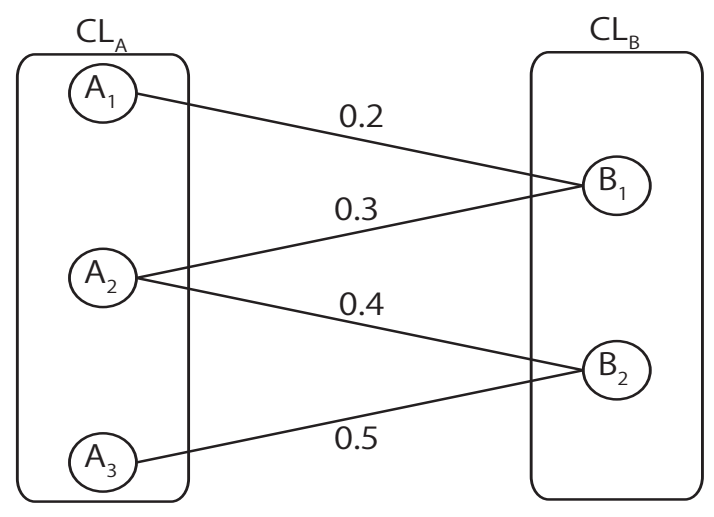

Fig. 3 Example of RBC.

conducted. Through these studies, it is possible to analyze the following.

- group boundary

- number of members belonging to the group

- relationship among members within the group

However, the analytical method so far has analyzed individuals. Therefore, the relationship between individuals and individuals has been clearly analyzed, but the relationship between groups and individuals or between groups has not been clarified.

Therefore, in the fuzzy graph, a group is replaced as one node (hereafter referred to as a cluster node).

At this time, it is the number of elements (cluster size) $\left|C L_{A}\right| \geq 2$ of the cluster $C L_{A}$. Therefore, to define the relationship between a node and a cluster node, it is necessary to aggregate the relationships in two or more existing nodes into one. There are various methods for aggregating two or more relationships. For example, there is the method of taking the average of the relationships between all the nodes belonging to the cluster node and the analysis target node.

When aggregating based on the average, set arbitrary cluster nodes $C L_{A}$ and $C L_{B}$, the set of node pairs belonging to each 


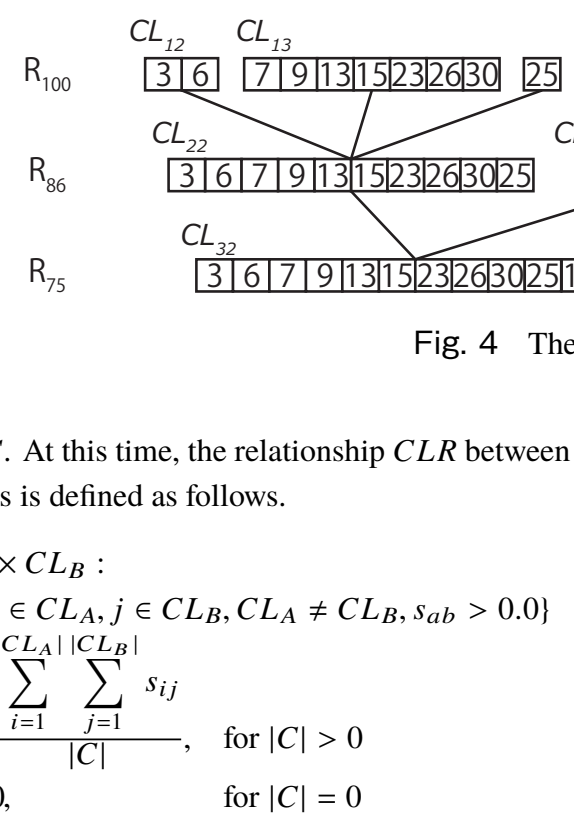

Similarly, the relationship $C I R$ of any cluster node $C L_{A}$ and node $j$ can also be defined as follows.

$$
\begin{aligned}
& C=C L_{A} \times j:\left\{(a, j) \mid a \in C L_{A}, s_{i j}>0.0\right\} \\
& C I R= \begin{cases}\frac{\sum_{i=1}^{\left|C L_{A}\right|} s_{i j}}{|C|}, & \text { for }|C|>0 \\
0, & \text { for }|C|=0\end{cases}
\end{aligned}
$$

\subsection{Relationship Analysis for Groups Belong- ing to Different Similarity Levels}

In human relationship analysis applying fuzzy graph, a partition tree is used. In the partition tree, it is clustered for each similarity level. The existing analysis method is relation analysis of nodes belonging to the same similarity level. When the similarity level of the partition tree changes, the cluster structure also changes according to the similarity level. When analyzing the relationship between clusters, one cluster size may be too large or too small. Therefore, by analyzing two clusters belonging to different similarity levels, it is possible to analyze them with the same cluster size. Let clusters to be analyzed be $C L_{A}$, $C L_{B}\left(A_{R} \cup B_{R^{\prime}}=\phi\right)$, cluster similarity levels $R, R^{\prime}$. At this time, the relationship between the two clusters can be calculated by the mathematical formula equivalent to the mathematical expression defined by 10 .

\section{Case Study}

In this section, we analyze by fuzzy graph model based on question survey result [13] from 30 students.

\subsection{Question Survey}

For the 30 students who are the subjects of the survey, the following question survey was conducted.

- Please write your $N$ friends in the order of those with whom
22

22
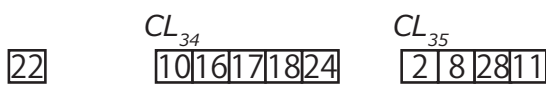

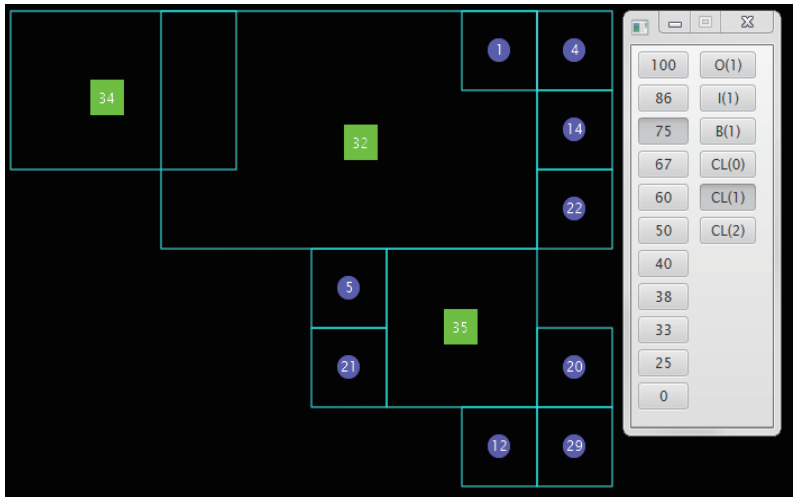

Fig. 5 Fuzzy graph (similarity level $=0.75)$.

(A1) you would like to study in group

(A2) you would like to take lunch together

(A3) you would like to do club activities

(A4) you would like to do volunteer activities

The partition tree created from the response matrix from the above question survey results is shown in the Fig. 2. Fig. 4 shows the extracted clusters to be analyzed in this section from Fig. 2. Fig. 4, $C L_{x}$ represents the cluster ID.

Also shown in Fig. 5 is a fuzzy graph showing similarity level $R=0.75$ based on Fig. 2. Squares represent cluster nodes (subgroup), and circles represent nodes (individuals).

\subsection{Results of Analysis}

In this section, the results of analyzing the following relationships from the question survey with 30 students are shown.

- Relationship between subgroups

- Relationship between subgroups belonging to different similarity levels

- Relationship between subgroup and individual

\subsection{Relationships between Subgroups}

Figure 6 shows the result of analyzing and displaying the relationship between subgroups 32 and 34 at similarity level 0.75 . The analysis result shows the average of the relationships of individuals belonging to the two subgroups. The relationship between the subgroups 32 and 34 is 0.52 , which means that they do not have particularly good relations.

\subsubsection{Relationship between Subgroups Be- longing to Different Similarity Levels}

The similarity level of subgroup 32 is changed from 0.75 to 


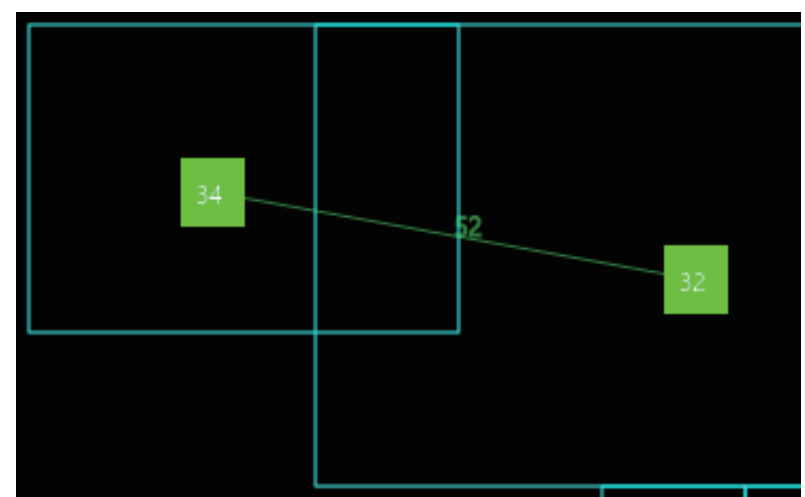

Fig. 6 The relationship between the subgroups 32 and 34 (similarity level $=0.75$ ).

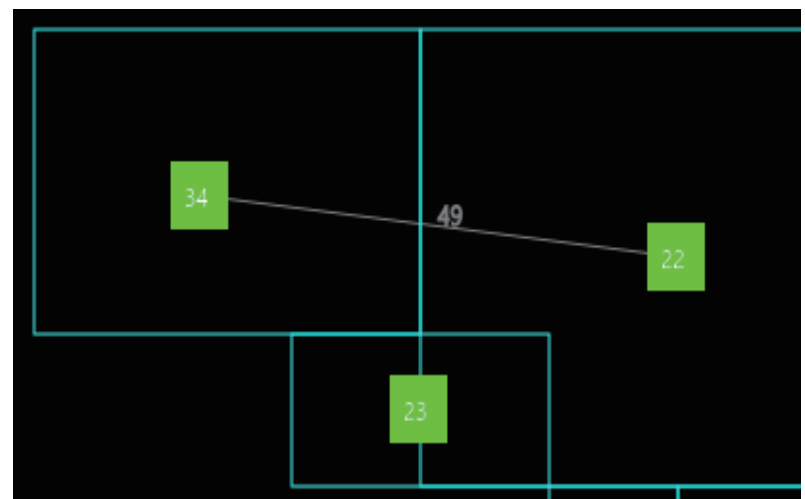

Fig. 7 The relationship between subgroup 22 (similarity level $=0.86$ ) and 34 (similarity level $=0.75$ ).

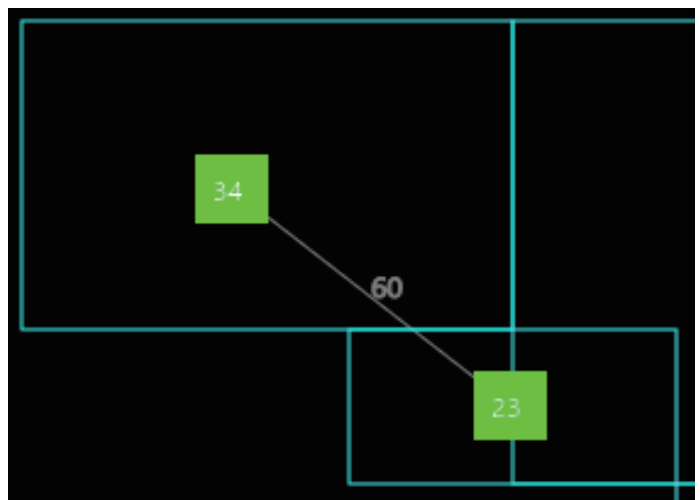

Fig. 8 The relationship between subgroup 23 (similarity level $=0.86)$ and 34 (similarity level $=0.75$ ).

0.86 . As a result, subgroup 32 is divided into two subgroups, 22 and 23. Fig. 7 shows a result of analyzing and displaying the relationship between subgroup 22 belonging to the similarity level of 0.86 and subgroup 34 belonging to the similarity level of 0.75 . Similarly, the relationship between subgroups 23 and 34 is shown in Fig. 8 .

Analysis result for subgroups 22 and 34 is 0.49 . On the other hand, the analysis result of subgroups 23 and 34 is 0.60 .

By dividing the subgroup, it is possible to distinguish between a better subgroup and a subgroup that is not better.

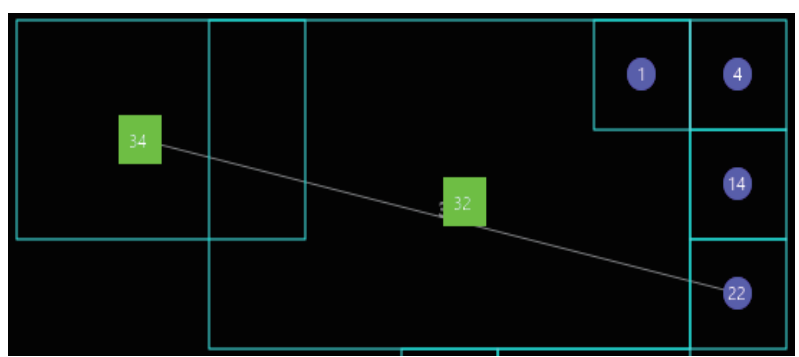

Fig. 9 Relationship between subgroup 34 (similarity level = 0.75 ) and individual 22.

\subsubsection{Relationship between Subgroup and In- dividual}

The result of analyzing the relationship between subgroup 34 and individual 22 is shown in Fig. 9. As a result of aggregating the relationship with each individual belonging to the subgroup using an average, the relation is 0.33 .

\subsection{Consideration}

As a result of analysis in Section 5.2.1, the relationship is 0.52 . However, when the similarity level is changed from 0.75 to 0.86 , the relationship with subgroup 23 is 0.60 . The cluster size of subgroup 32 is 12 . On the other hand, the cluster size of subgroup 34 is 5 . Therefore, when analyzing with subgroup 34 , there were also members with very good friends and members who were not such good friends. By changing the similarity level, the difference in size of the cluster to be analyzed can be made smaller. Therefore, it is possible to analyze clearly which subgroup has an intimate relationship in more detail. Also, the relationship between subgroups and individuals has been analyzed depending on analysts to this point. This analysis result showed that it can analyze quantitatively using an aggregating the relationship by average.

\section{Concluding Remarks}

We defined relationships on nodes and clusters belonging to different similarities and verified their effectiveness. Furthermore, we showed various analytical indices, and some of them were expanded to indices adapted to fuzzy graphs. In some indices, we defined using both directed graphs and undirected graphs, making them selectable according to the purpose of analysis. On the other hand, our proposed method focuses on analysis, and does not consider the intersection of edges and labels in drawing. Therefore, visibility may be severely impaired, for example, when the number of analysis targets increases. In future studies, we will propose a drawing algorithm for placement of edges and labels and an application for mapping data of multi-layers applying the fuzzy graph.

\section{References}

[1] J. L. Moreno. The Sociometry Reader. The Free Press, 1960.

[2] E. Tsuda, Y. Katsumata, H. Yamashita, and S. Shimizu. An application of fuzzy graph to sociometry analysis. Journal of Japan Society for Fuzzy Theory and Systems, 6(3):570-584, 1994.

[3] S. Shimizu and H. Yamashita. Approximate graphical analysis 
of fuzzy sociogram. Biomedical fuzzy and human sciences : the official journal of the Biomedical Fuzzy Systems Association, 1(1):43-47, 1995.

[4] H. Yamashita, T. Takizawa, H. Uesu, Y. Okuda, S. Kanagawa, S. Shimizu, J. Sho, K. Shinkai, M. Tomita, E. Tsuda, K. Nagashima, Y. Hashiguchi, K. Motegi, and M. Morioka. Introduction to Fuzzy Theory and Its Application. Kyoritsu-Shuppan, 2010.

[5] H. Uesu. Structure analysis of fuzzy node fuzzy graph and its application. Biomedical Soft Computing and Human Science, 11(1):41-49, 2006

[6] K. Shinkai. Fuzzy cluster analysis and its evaluation method. Biomedical Soft Computing and Human Science, 13(2):3-9, 2008.

[7] Y. Katsumata, E. Tsuda, K. Nagashima, and H. Yamashita. Opinion survey applying fuzzy graph. In Joint 9th IFSA World Congress and 20thNAFIPS International Conference, 131-134, 2012.

[8] W. D. Nooy, A. Mrvar, and V. Batagelj. Exploratory Social Network Analysis with Pajek. Cambridge University Press, 2011.

[9] M. Sumida. The Sociological Study of Child' s Peer Group. Kyushu University Press, 1995.

[10] T. Sumiya, S. Yamamoto, S. Kaneda, and H. Haga. Interpersonal network extraction based on non-verbal communication by using motion picture processing. IPSJ SIG Technical Report, 2012-CDS-3(4):1-9, 2012.

[11] A. Kuz, M. Falco, E. de Renzis, L. Nahuel, and R. Giandini. Using social network analysis in the classroom: a case study applying nodexl. In XXI Congreso Argentino de Ciencias de la Computación (Junín, 2015), 2015.

[12] T. Yoshizumi, T. Sumida, Y. Shiono, M. Namekawa, and Kensei Tsuchida. Analysis method for human relations through the touch interface. The Transactions of Human Interface Society, 17(2):151-158, 2015

[13] Y. Shiono, T. Kirishima, Y. Ueda, and K. Tsuchida. Drawing algorithm for fuzzy graph using the partition tree. Journal of Advanced Computational Intelligence and Intelligent Informatics, 16(5):888-896, 2012.

$\begin{array}{rr}(2017 \text { 年 } 4 \text { 月 } 4 \text { 日 } & \text { 受付) } \\ \text { (2017 年 } 5 \text { 月 } 10 \text { 日 } & \text { 採録) }\end{array}$

[Contact Address]

Graduate School of Engineering, Toyo University

2100 Kujirai, Kawagoe-shi, Saitama 350-8585, Japan

Toshihiro YOSHIZUMI

E-mail: s46d01310027@toyo.jp
Information about Author

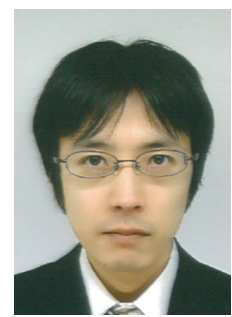

\section{Toshihiro YOSHIZUMI [Non-member]}

Received the M.E. degrees from Toyo University in 2009 . He is currently a doctoral course student of graduate school of Engineering, Toyo University, Japan. His research interests include application of graph algorithms, graph grammars and table processing systems.

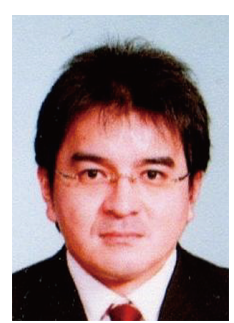

\section{Tomoo SUMIDA [Non-Member]}

Graduated with a Doctor of Engineering from Toyo University in 2009 . He has been a parttime Lecturer of Toyo University since 2009. His main research interests are network protocol, graph visualization, and software development. He is a member of IPSJ, IEICE Japan and IEEE.

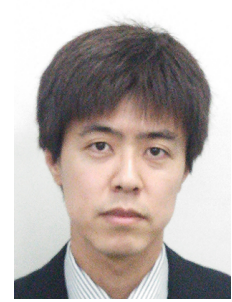

\section{Yasunori SHIONO [Non-member]}

Received the M.E. and Dr.Eng. degrees from Toyo University in 2006 and 2010 respectively. $\mathrm{He}$ is currently an assistant professor of the organization for information strategy and promotion, Yokohama National University, Japan. His research interests include application of graph algorithms, graph grammars and fuzzy theory.

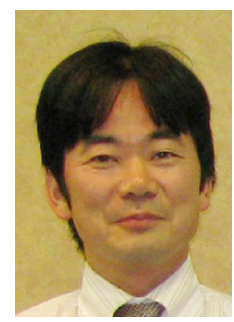

\section{Mitsuhiro NAMEKAWA [Member]}

Received the M.E. and Dr.Eng. degrees from Toyo University in 1993 and 2000 respectively. He is currently a professor of graduate school of business innovation, Kaetsu University, Japan. His research interests include application of system simulation, image recognition and fuzzy theory.

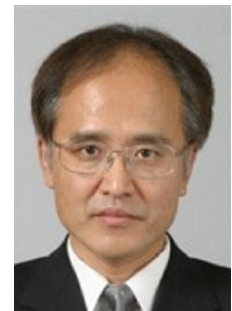

Kensei TSUCHIDA [Non-member]

Received M.S. and D.S. degrees in mathematics from Waseda University in 1984 and 1994 respectively. He is currently professor of faculty of information sciences and arts, Toyo University, Japan. His research interests include application of software visualization, human interface, graph language and graph algorithms. 